J. Korean Math. Soc. 50 (2013), No. 1, pp. 203-218

http://dx.doi.org/10.4134/JKMS.2013.50.1.203

\title{
ON GI-FLAT MODULES AND DIMENSIONS
}

\author{
Zenghui GaO
}

\begin{abstract}
Let $R$ be a ring. A right $R$-module $M$ is called GI-flat if $\operatorname{Tor}_{1}^{R}(M, G)=0$ for every Gorenstein injective left $R$-module $G$. It is shown that GI-flat modules lie strictly between flat modules and copure flat modules. Suppose $R$ is an $n$-FC ring, we prove that a finitely presented right $R$-module $M$ is GI-flat if and only if $M$ is a cokernel of a Gorenstein flat preenvelope $K \rightarrow F$ of a right $R$-module $K$ with $F$ flat. Then we study GI-flat dimensions of modules and rings. Various results in [6] are developed, some new characterizations of von Neumann regular rings are given.
\end{abstract}

\section{Introduction}

Throughout this paper, $R$ is an associative ring with identity and all modules are unitary. For any $R$-module $M$, we use $\mathrm{fd}_{R}(M), \operatorname{id}_{R}(M)$ and $\mathrm{FP}_{-i d}(M)$ to denote the flat, injective and FP-injective dimensions of $M$, respectively. The character module $\operatorname{Hom}_{\mathbb{Z}}\left(M, \mathbb{Q} / \mathbb{Z}\right.$ ) is denoted by $M^{+}$. We use $l . D(R)$ (resp., $w D(R))$ to stand for the left global dimension (resp., weak global dimension) of a ring $R$. For unexplained concepts and notations, we refer the reader to $[11,17,19]$.

Let $R$ be a ring. A right $R$-module $M$ is called copure flat if $\operatorname{Tor}_{1}^{R}(M, E)=0$ for all injective left $R$-modules $E$, and $M$ is said to be strongly copure flat provided that $\operatorname{Tor}_{i}^{R}(M, E)=0$ for all injective left $R$-modules $E$ and all $i \geqslant 1$. These modules were introduced and studied by Enochs and Jenda in [9]. Many properties of these modules were given by Enochs, Jenda, Sazeedeh, Ding and Chen etc in $[7,9,11,20]$. In [9], the copure flat dimension, $\operatorname{cfd}(M)$, of a right $R$-module $M$ is defined to be the largest positive integer $n$ such that $\operatorname{Tor}_{n}^{R}(M, E) \neq 0$ for some injective left $R$-module $E$. The copure flat modules together with the copure flat dimension in [9] were used to characterize $n$ Gorenstein rings (i.e., $R$ is left and right Noetherian with $\operatorname{id}\left({ }_{R} R\right) \leqslant n$ and $\operatorname{id}\left(R_{R}\right) \leqslant n$ for an integer $\left.n \geqslant 0\right)$.

Received March 20, 2012; Revised July 12, 2012.

2010 Mathematics Subject Classification. 16E30, 16E10, 16E50.

Key words and phrases. Gorenstein injective module, GI-flat module, GI-flat dimension, $n$-FC ring, Gorenstein flat preenvelope. 
Recall a left $R$-module $M$ is called Gorenstein injective [10] if there exists an exact sequence $\cdots \rightarrow E_{1} \rightarrow E_{0} \rightarrow E^{0} \rightarrow E^{1} \rightarrow \cdots$ of injective left $R$-modules with $M=\operatorname{ker}\left(E^{0} \rightarrow E^{1}\right)$ such that $\operatorname{Hom}_{R}(E,-)$ leaves the sequence exact whenever $E$ is an injective left $R$-module. A left $R$-module $M$ is said to be Gorenstein flat [13] if there is an exact sequence $\cdots \rightarrow F_{1} \rightarrow F_{0} \rightarrow F^{0} \rightarrow F^{1} \rightarrow$ $\cdots$ of flat left $R$-modules with $M=\operatorname{ker}\left(F^{0} \rightarrow F^{1}\right)$ such that $I \otimes_{R}-$ leaves the sequence exact whenever $I$ is an injective right $R$-module. Gorenstein injective and flat modules have been studied by many authors (see, for example, $[2,4,8,10,13,11,17,21])$. The Gorenstein injective and flat dimensions of an $R$-module $M$, denoted by $\operatorname{Gid}_{R}(M)$ and $\operatorname{Gfd}_{R}(M)$, are defined in terms of resolutions by Gorenstein injective and flat modules, respectively. Bennis and Mahdou in [2, Example 2.5] showed that Gorenstein injective modules are not necessarily projective (flat) over QF-rings. More recently, the author in [16] introduced the notion of GI-injective modules and discussed the GI-injective dimension of modules and rings. One easily checks that every Gorenstein injective left $R$-module is flat implies $R$ is left IF (i.e., rings satisfying every injective left $R$-module is flat [5]), but the converse is not true in general. Inspired by $[9,16]$, in this paper, we will introduce a concept of GI-flat modules and characterize the GI-flat dimension of modules and rings.

In Section 2, we give the definition of GI-flat modules and show some of the general properties. It is shown that GI-flat modules situate between flat modules and copure flat modules (see Remark 2.2). For any ring $R$, it is proved that a right $R$-module $M$ is flat if and only if $M$ is GI-flat and $\operatorname{Gfd}_{R}(N) \leqslant 1$. Let $R$ be an $n$-FC ring (i.e., $R$ is left and right coherent with self-FP-injective dimension at most $n$ on either side [8]). We prove that a finitely presented right $R$-module $M$ is GI-flat if and only if $M$ is a cokernel of a Gorenstein flat preenvelope $K \rightarrow F$ of a right $R$-module $K$ with $F$ flat (Theorem 2.11).

In Section 3, we characterize the right global GI-flat dimension, $r . G I F D(R)$, of a ring $R$ in terms of Gorenstein injective and flat modules. For any ring $R$, it is shown that (Theorem 3.4)

r.GIFD $(R)=\sup \left\{\operatorname{fd}_{R}(N) \mid N\right.$ is a Gorenstein injective left $R$-module $\}$.

Moreover, if $R$ is a commutative coherent ring with $G I F D(R)<\infty$, then

$$
\begin{aligned}
G I F D(R) & =\sup \left\{\operatorname{fd}_{R}(N) \mid N \text { is a Gorenstein injective } R \text {-module }\right\} \\
& =\sup \left\{\mathrm{FP}-\mathrm{id}_{R}(M) \mid M \text { is a Gorenstein flat } R \text {-module }\right\} .
\end{aligned}
$$

Suppose $R$ is an $n$-FC ring. We have the following equalities (Proposition 3.9)

$$
\begin{aligned}
r . G I F D(R) & =\sup \left\{\operatorname{fd}_{R}(M) \mid M \text { is a Gorenstein injective left } R \text {-module }\right\} \\
& =\sup \left\{\operatorname{fd}_{R}(M) \mid M \text { is a left } R \text {-module with } \operatorname{Gid}_{R}(M)<\infty\right\} \\
& =\sup \left\{\operatorname{fd}_{R}\left(N^{+}\right) \mid N \text { is a right } R \text {-module with } \operatorname{Gfd}_{R}(N)<\infty\right\} \\
& =\sup \left\{\mathrm{FP}_{-}-\mathrm{id}_{R}(N) \mid N \text { is a Gorenstein flat right } R \text {-module }\right\} \\
& =\sup \left\{\mathrm{FP}-\mathrm{id}_{R}(N) \mid N \text { is a right } R \text {-module with } \operatorname{Gfd}_{R}(N)<\infty\right\} .
\end{aligned}
$$


Moreover, for any ring $R$, we prove that $\operatorname{r} \cdot \operatorname{GIFD}(R) \leqslant 1$ if and only if every submodule of a projective right $R$-module is GI-flat if and only if every submodule of a (GI-)flat right $R$-module is GI-flat (Proposition 3.12). If $R$ is a left coherent ring, then $R$ is left semihereditary if and only if $r \cdot G I F D(R) \leqslant 1$ and every GI-flat right $R$-module is flat. We also give some new descriptions of von Neumann regular rings (Proposition 3.16 and Corollary 3.17).

\section{GI-flat modules}

In this section we give a treatment of GI-flat modules. It is shown that GIflat modules lie strictly between flat modules and copure flat modules. Some general properties of GI-flat modules are also discussed.

Definition 2.1. A right $R$-module $M$ is called $G I$-flat if $\operatorname{Tor}_{1}^{R}(M, G)=0$ for all Gorenstein injective left $R$-modules $G$.

Remark 2.2. (1) Since every injective module is Gorenstein injective, we have the following inclusions:

$\{$ flat modules $\} \subseteq\{$ GI-flat modules $\} \subseteq\{$ copure flat modules $\}$.

(2) From [1, Lemma 2.5], we can deduce easily that over rings with finite weak global dimension, the class of all flat modules coincides with the one of all copure flat modules and then with the one of all GI-flat modules.

(3) To give an example of copure flat module which is not GI-flat it is sufficient to use Proposition 3.16 and the fact that a commutative ring $R$ is an IF-ring if and only if every $R$-module is copure flat.

(4) Also, using Proposition 2.6, we can show that there is a GI-flat module which is not flat.

Recall a left $R$-module $M$ is called GI-injective [16] provided that

$$
\operatorname{Ext}_{R}^{1}(N, M)=0
$$

for all Gorenstein injective left $R$-modules $N$. One easily gets that a right $R$-module $M$ is GI-flat if and only if $M^{+}$is GI-injective by the standard isomorphism: $\operatorname{Ext}_{R}^{1}\left(N, M^{+}\right) \cong \operatorname{Tor}_{1}^{R}(M, N)^{+}$for any left $R$-module $N$.

Proposition 2.3. The class of GI-flat modules is closed under extensions. Moreover, if $\left(M_{i}\right)_{i \in I}$ is a family of right $R$-modules, then $\bigoplus M_{i}$ is GI-flat if and only if each $M_{i}$ is GI-flat.

Proof. The first assertion follows immediately by definition, and the second assertion holds by the isomorphism: $\operatorname{Tor}_{1}^{R}\left(\bigoplus M_{i}, N\right) \cong \bigoplus \operatorname{Tor}_{1}^{R}\left(M_{i}, N\right)$ for all left $R$-modules $N$.

Proposition 2.4. Every pure submodule of a GI-flat right R-module is GI-flat.

Proof. Let $M$ be a GI-flat right $R$-module and $N$ a pure submodule of $M$. The pure exact sequence $0 \rightarrow N \rightarrow M \rightarrow M / N \rightarrow 0$ induces a split exact sequence 
$0 \rightarrow(M / N)^{+} \rightarrow M^{+} \rightarrow N^{+} \rightarrow 0$. It is clear that $M^{+}$is GI-injective. Thus $N^{+}$ is GI-injective by [16, Proposition 2.3], and hence $N$ is GI-flat, as desired.

In general, GI-flat modules need not be flat as shown by the following result.

Proposition 2.5. Let $R$ be a ring. Then a right $R$-module $M$ is flat if and only if $M$ is GI-flat and $\operatorname{Gfd}_{R}(M) \leqslant 1$.

Proof. "Only if" part is by definition.

"If " part. Let $M$ be a GI-flat right $R$-module and $\operatorname{Gfd}_{R}(M) \leqslant 1$. Then $\operatorname{Gid}\left(M^{+}\right) \leqslant 1$ by [17, Proposition 3.11]. There exists an exact sequence $0 \rightarrow$ $M^{+} \rightarrow E \rightarrow L \rightarrow 0$, where $E$ is injective and $L$ is Gorenstein injective by $\left[17\right.$, Theorem 2.22]. It follows that $\operatorname{Ext}_{R}^{1}\left(L, M^{+}\right)=0$ since $M^{+}$is GI-injective. Thus the sequence $0 \rightarrow M^{+} \rightarrow E \rightarrow L \rightarrow 0$ is split, and so $M^{+}$is injective. Therefore $M$ is flat, as desired.

Proposition 2.6. The following are equivalent for a right $R$-module $M$ :

(1) $M$ is GI-flat.

(2) $M \in{ }^{\perp} \mathcal{C}$, where $\mathcal{C}=\left\{B^{+} \mid B\right.$ is a Gorenstein injective left $R$-module $\}$ and ${ }^{\perp} \mathcal{C}=\left\{L \mid \operatorname{Ext}_{R}^{1}(L, C)=0\right.$ for all $\left.C \in \mathcal{C}\right\}$.

(3) For every exact sequence $0 \rightarrow A \rightarrow B \rightarrow C \rightarrow 0$ of left $R$-modules, where $C$ is Gorenstein injective, the functor $M \otimes_{R}-$ preserves the exactness.

Proof. (1) $\Leftrightarrow(2)$ follows from the standard isomorphism:

$$
\operatorname{Tor}_{1}^{R}(M, N)^{+} \cong \operatorname{Ext}_{R}^{1}\left(M, N^{+}\right)
$$

for any left $R$-module $N$ by [3, Chapter VI. Proposition 5.1].

$(1) \Rightarrow(3)$ is by definition.

$(3) \Rightarrow(1)$ For any Gorenstein injective left $R$-module $N$, there is a short exact sequence $0 \rightarrow K \rightarrow F \rightarrow N \rightarrow 0$, where $F$ is flat. Then we have the exact sequence

$$
0 \rightarrow \operatorname{Tor}_{1}^{R}(M, N) \rightarrow M \otimes_{R} K \rightarrow M \otimes_{R} F \rightarrow M \otimes_{R} N \rightarrow 0 .
$$

But $0 \rightarrow M \otimes_{R} K \rightarrow M \otimes_{R} F \rightarrow M \otimes_{R} N \rightarrow 0$ is exact by (3). Therefore $\operatorname{Tor}_{1}^{R}(M, N)=0$, and so $M$ is GI-flat.

For a positive integer $n$, a left $R$-module $A$ is called an $n$-syzygy module (of a left $R$-module $M)$ if there is an exact sequence $0 \rightarrow A \rightarrow P_{n-1} \rightarrow \cdots \rightarrow$ $P_{1} \rightarrow P_{0} \rightarrow M \rightarrow 0$ with all $P_{i}$ projective.

Proposition 2.7. Let $R$ be a ring. If $\operatorname{Tor}_{i}^{R}(M, G)=0$ for any $i$ with $1 \leqslant i \leqslant$ $n+1$ and any Gorenstein injective left $R$-module $G$, then every $k$ th syzygy of $M$ is GI-flat for $0 \leqslant k \leqslant n$.

Proof. Let $k$ be an integer with $0 \leqslant k \leqslant n$, and $C_{k}$ a $k$ th syzygy of $M$. Then there exists an exact sequence

$$
0 \rightarrow C_{k} \rightarrow P_{k-1} \rightarrow \cdots \rightarrow P_{1} \rightarrow P_{0} \rightarrow M \rightarrow 0,
$$


where each $P_{i}(0 \leqslant i \leqslant k-1)$ is projective. For every Gorenstein injective left $R$-module $G$, one easily checks that $\operatorname{Tor}_{1}^{R}\left(C_{k}, G\right) \cong \operatorname{Tor}_{k+1}^{R}(M, G)$. Note that $\operatorname{Tor}_{k+1}^{R}(M, G)=0$ by the hypothesis, it follows that $\operatorname{Tor}_{1}^{R}\left(C_{k}, G\right)=0$, and so $C_{k}$ is GI-flat.

We will say a right $R$-module $M$ strongly GI-flat if $\operatorname{Tor}_{i}^{R}(M, E)=0$ for all Gorenstein injective left $R$-modules $E$ and all $i \geqslant 1$.

Proposition 2.8. Let $R$ be an $n$-Gorenstein ring. Then the following are equivalent for a right $R$-module $M$ :

(1) $M$ is strongly GI-flat.

(2) $\operatorname{Tor}_{1}^{R}(M, N)=0$ for all left $R$-modules $N$.

(3) $\operatorname{Tor}_{i}^{R}(M, N)=0$ for all left $R$-modules $N$ and all $i \geqslant 1$.

Proof. (1) $\Rightarrow(2)$ Suppose $N$ is a left $R$-module, then $\operatorname{Gid}_{R}(N) \leqslant n$ by [11, Theorem 12.3.1] since $R$ is $n$-Gorenstein. So there exists an exact sequence

$$
0 \rightarrow N \rightarrow E_{0} \rightarrow E_{1} \rightarrow \cdots \rightarrow E_{m} \rightarrow 0
$$

with each $E_{i}$ Gorenstein injective by definition. Let $L_{0}=N, L_{i}=\operatorname{im}\left(E_{i-1} \rightarrow\right.$ $\left.E_{i}\right)$ for $1 \leqslant i \leqslant m-1$ and $L_{m}=E_{m}$. Then

$$
0 \rightarrow L_{i} \rightarrow E_{i} \rightarrow L_{i+1} \quad \text { for } 1 \leqslant i \leqslant m-1
$$

are exact. Therefore, by (1), we have

$$
\operatorname{Tor}_{1}^{R}(M, N) \cong \operatorname{Tor}_{2}^{R}\left(M, L_{1}\right) \cong \cdots \cong \operatorname{Tor}_{m+1}^{R}\left(M, E_{m}\right)=0 .
$$

$(2) \Rightarrow(3)$ Let $N$ be a left $R$-module. Then $\operatorname{Gid}_{R}(N) \leqslant n$ by [11, Theorem 12.3.1]. Next we will prove that $\operatorname{Tor}_{i}^{R}(M, N)=0$ for all $i \geqslant 1$. We proceed by induction on $i$. The case $i=1$ is trivial. Let $i \geqslant 2$ and choose a partial projective resolution of $N$ :

$$
0 \rightarrow K_{i-1} \rightarrow P_{i-2} \rightarrow \cdots \rightarrow P_{0} \rightarrow N \rightarrow 0 .
$$

Then $\operatorname{Tor}_{i}^{R}(M, N) \cong \operatorname{Tor}_{1}^{R}\left(M, K_{i-1}\right)=0$ by (2). Thus (3) follows.

(3) $\Rightarrow$ (1) It follows from (3) that $M$ is flat by [19, Theorem 9.13]. The desired result follows from the fact that every flat module is strongly GI-flat.

Let $\mathcal{F}$ be a class of $R$-modules and $M$ an $R$-module. Following [11], we say that a homomorphism $f: M \rightarrow F$ is an $\mathcal{F}$-preenvelope if $F \in \mathcal{F}$ and the abelian group homomorphism $\operatorname{Hom}_{R}\left(f, F^{\prime}\right): \operatorname{Hom}_{R}\left(F, F^{\prime}\right) \rightarrow \operatorname{Hom}_{R}\left(M, F^{\prime}\right)$ is surjective for each $F^{\prime} \in \mathcal{F}$. It has been shown that every right (or left) $R$-module has a Gorenstein flat preenvelope over any $n$-FC ring ([18, Theorem $5.3])$.

Lemma 2.9. Let $R$ be an $n-F C$ ring. If $M$ is a cokernel of a Gorenstein flat preenvelope $f: K \rightarrow F$ of a right $R$-module $K$ with $F$ flat, then $M$ is GI-flat. 
Proof. Let $M$ be a cokernel of a Gorenstein flat preenvelope $f: K \rightarrow F$ of a right $R$-module $K$ with $F$ flat. Let $L=\operatorname{im}(f)$, then $0 \rightarrow L \rightarrow F \rightarrow M \rightarrow 0$ is exact and $L \rightarrow F$ is a Gorenstein flat preenvelope of $L$. For every Gorenstein injective left $R$-module $E$, we have $E^{+}$is Gorenstein flat by [8, Proposition 12] since $R$ is $n$-FC. So we get the exactness of $\operatorname{Hom}_{R}\left(F, E^{+}\right) \rightarrow \operatorname{Hom}_{R}\left(L, E^{+}\right) \rightarrow$ 0 , which induces the exactness of the sequence $\left(F \otimes_{R} E\right)^{+} \rightarrow\left(L \otimes_{R} E\right)^{+} \rightarrow 0$. Thus the sequence $0 \rightarrow L \otimes_{R} E \rightarrow F \otimes_{R} E$ is exact. Note that $F$ is flat, it follow that $0 \rightarrow \operatorname{Tor}_{1}^{R}(M, E) \rightarrow L \otimes_{R} E \rightarrow F \otimes_{R} E$ is exact, and hence $\operatorname{Tor}_{1}^{R}(M, E)=0$. Thus $M$ is GI-flat.

Lemma 2.10. Let $R$ be an $n-F C$ ring and $M$ a finitely presented right $R$ module. If $M$ is GI-flat, then $M$ is a cokernel of a Gorenstein flat preenvelope $K \rightarrow F$ of a right $R$-module $K$ with $F$ flat.

Proof. Suppose $M$ is a finitely presented right $R$-module, then there exists an exact sequence $0 \rightarrow K \rightarrow P \rightarrow M \rightarrow 0$, where $P$ is finitely generated projective and $K$ is finitely generated. Next we will prove that $K \rightarrow P$ is a Gorenstein flat preenvelope. For any Gorenstein flat right $R$-module $F$, we have $F^{+}$is Gorenstein injective by [17, Theorem 3.6] (or [8, Theorem 5]), and so $\operatorname{Tor}_{1}^{R}\left(M, F^{+}\right)=0$ since $M$ is GI-flat. Thus we get the exact commutative diagram:

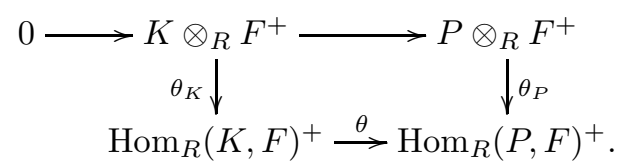

On the other hand, since $K$ is finitely generated, there exists an exact sequence $Q \rightarrow K \rightarrow 0$ with $Q$ finitely generated projective. Then the sequence $0 \rightarrow \operatorname{Hom}_{R}(K, F) \rightarrow \operatorname{Hom}_{R}(Q, F)$ is exact. So we have the following exact commutative diagram:

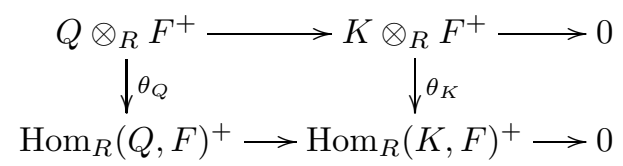

Note that $\theta_{Q}$ is an isomorphism by [19, Lemma 3.59], and so $\theta_{K}$ is epic. Since $\theta_{P}$ is an isomorphism, it follows that $\theta$ is a monomorphism. Therefore the sequence $\operatorname{Hom}_{R}(P, F) \rightarrow \operatorname{Hom}_{R}(K, F) \rightarrow 0$ is exact, which shows that $K \rightarrow P$ is a Gorenstein flat preenvelope.

Combining Lemma 2.9 with Lemma 2.10, we immediately obtain the following result.

Theorem 2.11. Let $R$ be an $n-F C$ ring and $M$ a finitely presented right $R$ module. Then $M$ is GI-flat if and only if $M$ is a cokernel of a Gorenstein flat preenvelope $K \rightarrow F$ of a right $R$-module $K$ with $F$ flat. 


\section{The GI-flat dimensions of modules and rings}

In this section we investigate the GI-flat dimensions of modules and rings. Some results in [6] are developed. We start with the following definition.

Definition 3.1. Let $R$ be a ring. The GI-flat dimension, GI-fd ${ }_{R}(M)$, of a right $R$-module $M$ is defined to be the largest positive integer $n$ such that $\operatorname{Tor}_{n}^{R}(M, N) \neq 0$ for some Gorenstein injective left $R$-modules $N$. The right global GI-flat dimension r.GIFD $R$ ) of $R$ is defined as

$$
\text { r.GIFD }(R)=\sup \left\{\mathrm{GI}-\mathrm{fd}_{R}(M) \mid M \text { is a right } R \text {-module }\right\} .
$$

Similarly, we have l.GIFD $R$ ) (when $R$ is commutative, we drop the unneeded letters $r$ and $l$ ).

If $M$ is strongly GI-flat, that is, $\operatorname{Tor}_{i}^{R}(M, N)=0$ for all $i \geqslant 1$ and all Gorenstein injective left $R$-modules $N$, we set $\operatorname{GI}_{-}-f_{R}(M)=0$.

Remark 3.2. (1) Since every injective left $R$-module is Gorenstein injective, by $\left[9\right.$, Lemma 3.3], we have $\operatorname{cfd}(M) \leqslant \operatorname{GI}^{-f_{d}}(M) \leqslant \operatorname{fd}_{R}(M)$ for any right $R$-module $M$ and $r . G I F D(R) \leqslant w D(R)$.

(2) The GI-flat dimension for rings measures how far away a commutative ring is from being von Neumann regular ring by Proposition 3.16.

Proposition 3.3. Let $R$ be a ring. Then the following are equivalent for a right $R$-module $M$ :

(1) $G I-f d_{R}(M) \leqslant n$.

(2) $\operatorname{Tor}_{n+j}^{R}(M, N)=0$ for all Gorenstein injective left $R$-modules $N$ and all $j \geqslant 1$.

(3) For each exact sequence $0 \rightarrow K_{n} \rightarrow F_{n-1} \rightarrow \cdots \rightarrow F_{1} \rightarrow F_{0} \rightarrow M \rightarrow 0$ where $F_{0}, \ldots, F_{n-1}$ are flat, then $K_{n}$ is strongly GI-flat.

Proof. $(1) \Leftrightarrow(2)$ is by definition.

$(2) \Rightarrow(3)$ Let $0 \rightarrow K_{n} \rightarrow F_{n-1} \rightarrow \cdots \rightarrow F_{1} \rightarrow F_{0} \rightarrow M \rightarrow 0$ be exact with $F_{0}, F_{1}, \ldots, F_{n-1}$ flat. Let $L_{0}=M, L_{i}=\operatorname{ker}\left(F_{i-1} \rightarrow F_{i-2}\right)$ for $1 \leqslant i \leqslant n-1$ and $L_{n}=K_{n}$. Then

$$
0 \rightarrow L_{i+1} \rightarrow F_{i} \rightarrow L_{i} \rightarrow 0 \text { for } 0 \leqslant i \leqslant n-1
$$

are exact. For any Gorenstein injective left $R$-module $N$ and for all $j \geqslant 1$, it follows from (2) that

$$
\begin{aligned}
\operatorname{Tor}_{j}^{R}\left(K_{n}, N\right) & \cong \operatorname{Tor}_{j+1}^{R}\left(L_{n-1}, N\right) \cong \operatorname{Tor}_{j+2}^{R}\left(L_{n-2}, N\right) \\
& \cong \cdots \cong \operatorname{Tor}_{j+n}^{R}(M, N)=0 .
\end{aligned}
$$

Therefore $K_{n}$ is strongly GI-flat.

$(3) \Rightarrow(2)$ Let $\cdots \rightarrow F_{1} \rightarrow F_{0} \rightarrow M \rightarrow 0$ be a flat resolution of $M$, and $L_{n}=\operatorname{ker}\left(F_{n-1} \rightarrow F_{n-2}\right)$. Then we get an exact sequence

$$
0 \rightarrow L_{n} \rightarrow F_{n-1} \rightarrow \cdots \rightarrow F_{1} \rightarrow F_{0} \rightarrow M \rightarrow 0 .
$$


It follows from (3) that $L_{n}$ is strongly GI-flat. For any Gorenstein injective left $R$-module $N$, we have $\operatorname{Tor}_{j+n}^{R}(M, N) \cong \operatorname{Tor}_{j}^{R}\left(L_{n}, N\right)=0$ for all $j \geqslant 1$. Thus (2) follows.

The following result plays a crucial role in this section.

Theorem 3.4. Let $R$ be a ring. Then

(1) r.GIFD $(R)=\sup \left\{f d_{R}(N) \mid N\right.$ is a Gorenstein injective left $R$-module $\}$.

(2) If $R$ is a commutative coherent ring with $G I F D(R)<\infty$, then

$$
\begin{aligned}
\operatorname{GIFD}(R) & =\sup \left\{f d_{R}(N) \mid N \text { is a Gorenstein injective } R \text {-module }\right\} \\
& =\sup \left\{F P-i d_{R}(M) \mid M \text { is a Gorenstein flat } R \text {-module }\right\} .
\end{aligned}
$$

Proof. (1) First, we show

$$
\text { r.GIFD(R) } \leqslant \sup \left\{\operatorname{fd}_{R}(N) \mid N \text { is a Gorenstein injective left } R \text {-module }\right\} .
$$

We may suppose that $\sup \left\{\operatorname{fd}_{R}(N) \mid N\right.$ is a Gorenstein injective left $R$-module $\}$ $=m<\infty$. Let $M$ be a right $R$-module. Then we have $\operatorname{Tor}_{m+1}^{R}(M, N)=$ 0 for any Gorenstein injective left $R$-module $N$ since $\operatorname{fd}_{R}(N) \leqslant m$, and so $\operatorname{GI}^{-f_{R}}(M) \leqslant m$. Thus $r$. GIFD $(R) \leqslant m$.

Next we shall prove $\sup \left\{\operatorname{fd}_{R}(N) \mid N\right.$ is a Gorenstein injective left $R$-module $\}$ $\leqslant r$. GIFD $(R)$. In fact, we may assume that $r \cdot G I F D(R)=n<\infty$. For any right $R$-module $M$, it follows that $\operatorname{GI}-\mathrm{fd}_{R}(M) \leqslant n$. Let $N$ be a Gorenstein injective left $R$-module. Then $\operatorname{Tor}_{n+1}^{R}(M, N)=0$ by Proposition 3.3, which implies that $\operatorname{fd}_{R}(N) \leqslant n$, as desired.

(2) To show the following inequality holds

$$
\begin{aligned}
& \sup \left\{\operatorname{fd}_{R}(N) \mid N \text { is a Gorenstein injective } R \text {-module }\right\} \\
\leqslant & \sup \left\{\mathrm{FP}_{-} \operatorname{id}_{R}(M) \mid M \text { is a Gorenstein flat } R \text {-module }\right\},
\end{aligned}
$$

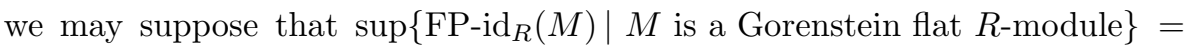
$m<\infty$. Let $N$ be a Gorenstein injective $R$-module. Then $\operatorname{fd}_{R}(N)=n<\infty$ by (1) since $\operatorname{GIFD}(R)<\infty$. We claim that $n \leqslant m$. Otherwise, let $n>m$. For any $R$-module $Q$, there exists an exact sequence $0 \rightarrow Q \rightarrow E \rightarrow L \rightarrow 0$ with $E$ injective, which induces the exactness of

$$
\operatorname{Tor}_{n+1}^{R}(L, N) \rightarrow \operatorname{Tor}_{n}^{R}(Q, N) \rightarrow \operatorname{Tor}_{n}^{R}(E, N) .
$$

Because every flat $R$-module is of FP-injective dimension at most $m$, we have $\mathrm{fd}_{R}(E) \leqslant m$ by [6, Theorem 3.8] since $R$ is coherent. Thus $\operatorname{Tor}_{n}^{R}(E, N)=0$ since $\mathrm{fd}_{R}(E) \leqslant m<n$, and $\operatorname{Tor}_{n+1}^{R}(L, N)=0$ since $\mathrm{fd}_{R}(N)=n$. It follows that $\operatorname{Tor}_{n}^{R}(Q, N)=0$, and so $\operatorname{fd}_{R}(N) \leqslant n-1$. This is a contradiction. Therefore $n \leqslant m$.

Now it remains to show the following inequality

$\sup \left\{\operatorname{fd}_{R}(N) \mid N\right.$ is a Gorenstein injective $R$-module $\}$ $\geqslant \sup \left\{\mathrm{FP}_{-\mathrm{id}}(M) \mid M\right.$ is a Gorenstein flat $R$-module $\}$. 
Since $\operatorname{GIFD}(R)<\infty$, by (1), we may assume that

$G I F D(R)=\sup \left\{\operatorname{fd}_{R}(N) \mid N\right.$ is a Gorenstein injective $R$-module $\}=n<\infty$.

It suffices to show that if $M$ is a Gorenstein flat $R$-module, then $\operatorname{FP}^{-i d}{ }_{R}(M) \leqslant$ $n$. The case $n=0$ holds by Proposition 3.16. Let $n \geqslant 1$. Assume first that $L$ is strongly Gorenstein flat. Then there exists a short exact sequence $0 \rightarrow L \rightarrow F \rightarrow L \rightarrow 0$ with $F$ flat by [2, Proposition 3.6]. By the Horseshoe Lemma ([19, Lemma 6.20]), we obtain the following commutative diagram:

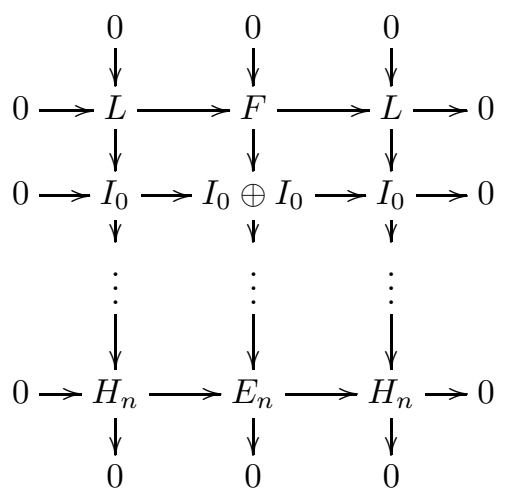

where $I_{i}$ is injective for $i=0,1, \ldots, n-1$. Note that every injective $R$-module is of flat dimension at most $n$ by hypothesis, which implies that $R$ is $n$-FC by [6, Theorem 3.8] since $R$ is commutative coherent. From the left vertical sequence, we get the exactness of the sequence

$$
0 \rightarrow\left(H_{n}\right)^{+} \rightarrow\left(I_{n-1}\right)^{+} \rightarrow \cdots \rightarrow\left(I_{0}\right)^{+} \rightarrow L^{+} \rightarrow 0,
$$

where $\left(I_{0}\right)^{+}, \ldots,\left(I_{n-1}\right)^{+}$are flat by $[15$, Theorem 2.2] since $R$ is coherent. From [8, Theorem 7], we get $\left(H_{n}\right)^{+}$is Gorenstein flat. On the other hand, since $\operatorname{GIFD}(R)=n$, we have GI-fd ${ }_{R}\left(L^{+}\right) \leqslant n$, and so $\left(H_{n}\right)^{+}$is strongly GI-flat by Proposition 3.3. So $\left(H_{n}\right)^{+}$is flat by Proposition 2.5, and thus $\operatorname{fd}_{R}\left(L^{+}\right) \leqslant n$. By [15, Theorem 2.2] again, we have $\mathrm{FP}_{-1} \mathrm{id}_{R}(L) \leqslant n$. Since every Gorenstein flat $R$-module is a direct summand of a strongly Gorenstein flat $R$-module by $\left[2\right.$, Theorem 3.5], which implies that $\operatorname{FP}-\mathrm{id}_{R}(M) \leqslant n$ for any Gorenstein flat $R$-module $M$. This completes the proof.

Proposition 3.5. Let $R$ be any ring. Then l.IFD $(R) \leqslant \operatorname{l.GIFD(R)\leqslant }$ $w D(R)$.

Proof. It suffices to prove l.IFD $(R) \leqslant$ l.GIFD $(R)$ by Theorem 3.4. We may assume that $l$.GIFD $(R)=n<\infty$. Let $M$ be an injective left $R$-module with $\operatorname{fd}_{R}(M)=m<\infty$. We claim that $m \leqslant n$. If not, suppose $m>n$. Let $N$ be a right $R$-module. Then there exists an exact sequence $0 \rightarrow N \rightarrow E(N) \rightarrow$ $E(N) / N \rightarrow 0$, which induces the following exact sequence

$$
\operatorname{Tor}_{m+1}^{R}(E(N) / N, M) \rightarrow \operatorname{Tor}_{m}^{R}(N, M) \rightarrow \operatorname{Tor}_{m}^{R}(E(N), M) .
$$


Then $\operatorname{Tor}_{m+1}^{R}(E(N) / N, M)=0$ since $\mathrm{fd}_{R}(M)=m$. Note that $\mathrm{fd}_{R}(E(N)) \leqslant$ $n<m$ by the hypothesis, it follows that $\operatorname{Tor}_{m}^{R}(E(N), M)=0$. Thus

$$
\operatorname{Tor}_{m}^{R}(N, M)=0
$$

for any right $R$-module $N$, and hence $\operatorname{fd}_{R}(M)<m$. This is a contradiction. So $m \leqslant n$, and the desired result follows.

Remark 3.6. (1) Using [5, Example 2], it is clear that the assertions "Every Gorenstein injective left $R$-module is flat" and "Every Gorenstein injective right $R$-module is flat" are not equivalent. In general, we have $\operatorname{r.GIFD}(R) \neq$ l.GIF $D(R)$ by Theorem 3.4 and Theorem 3.15.

(2) If $R$ is a ring with $l . D(R)<\infty$, then every Gorenstein injective left $R$-module is injective by the dual version of [17, Proposition 2.27], it follows that l.GIFD $(R)$ coincides with l.IFD $(R)$ by Theorem 3.4(1).

(3) Because Gorenstein injective modules need not be flat over a QF ring by [2, Example 2.5], and thus the left inequality in Proposition 3.5 may be strict.

Corollary 3.7. Let $R$ be any ring with $w D(R)<\infty$. Then

$$
r . G I F D(R)=\text { l.GIFD }(R)=w D(R) .
$$

Proof. This is a simple consequence of Proposition 3.5 and [6, Corollary 3.3].

We are now in a position to give some characterizations of $\operatorname{r.GIFD}(R)$, which is a generalization of [6, Theorem 3.5].

Theorem 3.8. For any ring $R$, we consider the following quantities:

(1) $r . G I F D(R)$.

(2) $\sup \left\{f d_{R}(M) \mid M\right.$ is a left $R$-module with $\left.\operatorname{Gid}_{R}(M)<\infty\right\}$.

(3) $\sup \left\{f d_{R}\left(N^{+}\right) \mid N\right.$ is a right $R$-module with $\left.\operatorname{Gfd}_{R}(N)<\infty\right\}$.

Then $(1)=(2) \geqslant(3)$. If $R$ is a left coherent ring, then $(1)=(2)=(3)$.

Proof. $(1) \leqslant(2)$ follows from Theorem 3.4(1).

$(2) \leqslant(1)$ We suppose that $r \cdot G I F D(R)=n<\infty$. For any left $R$-module $M$ with $\operatorname{Gid}_{R}(M)<\infty$, we may assume $\operatorname{Gid}_{R}(M)=m<\infty$. Then there is an exact sequence

$$
0 \rightarrow M \rightarrow E^{0} \rightarrow E^{1} \rightarrow \cdots \rightarrow E^{m} \rightarrow 0
$$

with each $E^{i}$ Gorenstein injective. It follows from $(2)$ that $\operatorname{fd}_{R}\left(E^{i}\right) \leqslant n$. Thus $\mathrm{fd}_{R}(M) \leqslant n$, and hence $(2) \leqslant(1)$ holds.

$(3) \leqslant(1)$ Assume that r.GIFD $(R)=n<\infty$. For any right $R$-module $N$ with $\operatorname{Gfd}_{R}(N)<\infty$, we may suppose $\operatorname{Gfd}_{R}(N)=k$. Then we have a Gorenstein flat resolution of $N$ :

$$
0 \rightarrow F_{k} \rightarrow \cdots \rightarrow F_{1} \rightarrow F_{0} \rightarrow N \rightarrow 0,
$$

which gives rise to the exactness of $0 \rightarrow N^{+} \rightarrow F_{0}^{+} \rightarrow F_{1}^{+} \rightarrow \cdots \rightarrow F_{k}^{+} \rightarrow$ 0 , where each $F_{i}^{+}$is Gorenstein injective by [17, Theorem 3.6]. Note that $\operatorname{fd}_{R}\left(F_{i}^{+}\right) \leqslant n$ by the hypothesis, whence $\operatorname{fd}_{R}\left(N^{+}\right) \leqslant n$, as desired. 
$(1) \leqslant(3)$ Let $R$ be left coherent and $(3)=n<\infty$. For any Gorenstein injective left $R$-module $M$, we have $M^{+}$is a right $R$-module. Then there exists a Gorenstein flat right $R$-module $F$ such that $F \rightarrow M^{+} \rightarrow 0$ is exact by [12] since $R$ is left coherent. This gives rise to the exactness of $0 \rightarrow M^{++} \rightarrow F^{+}$. It follows from [14, Proposition 3.52] that $0 \rightarrow M \rightarrow M^{++}$is exact, and so $0 \rightarrow M \rightarrow F^{+}$is exact. Thus $M$ is a direct summand of $F^{+}$and hence $\mathrm{fd}_{R}(M) \leqslant \operatorname{fd}_{R}\left(F^{+}\right) \leqslant n$. Therefore $(1) \leqslant(3)$ holds by Theorem $3.4(1)$.

For $n$-FC rings, we get the next result, which gives a generalization of $[6$, Theorem 3.8].

Proposition 3.9. The following are identical for an $n-F C$ ring $R$.

(1) r.GIFD $(R)$.

(2) $\sup \left\{f d_{R}(M) \mid M\right.$ is a left $R$-module with $\left.\operatorname{Gid}_{R}(M)<\infty\right\}$.

(3) $\sup \left\{f d_{R}\left(N^{+}\right) \mid N\right.$ is a right $R$-module with $\left.\operatorname{Gfd}_{R}(N)<\infty\right\}$.

(4) $\sup \left\{F P-i d_{R}(N) \mid N\right.$ is a Gorenstein flat right $R$-module $\}$.

(5) $\sup \left\{F P-i d_{R}(N) \mid N\right.$ is a right $R$-module with $\left.\operatorname{Gfd}_{R}(N)<\infty\right\}$.

Proof. $(1)=(2)=(3)$ follows from Theorem 3.8.

$(1) \leqslant(4)$ Let $M$ be a Gorenstein injective left $R$-module. Then $M^{+}$is Gorenstein flat by [8, Proposition 12] since $R$ is $n$-FC. It is easy to see that

$\mathrm{FP}_{-i d}\left(M^{+}\right) \leqslant \sup \left\{\mathrm{FP}-\mathrm{id}_{R}(N) \mid N\right.$ is a Gorenstein flat right $R$-module $\}$.

Note that $\operatorname{fd}_{R}(M)=\mathrm{FP}-\mathrm{id}_{R}\left(M^{+}\right)$by [15, Theorem 2.1$]$, it follows that $(1) \leqslant$ (4), as desired.

$(4) \leqslant(5)$ is trivial.

$(5) \leqslant(1)$ Let $M$ be a right $R$-module with $\operatorname{Gfd}_{R}(M)<\infty$. Then $\operatorname{fd}_{R}\left(M^{+}\right) \leqslant$ r.GIF $D(R)$ by Theorem 3.8. Hence FP-id $R(M)=\mathrm{fd}_{R}\left(M^{+}\right) \leqslant r . G I F D(R)$ by $[15$, Theorem 2.2$]$, whence $(5) \leqslant(1)$ follows.

Corollary 3.10. The following are identical for an $n$-Gorenstein ring $R$.

(1) $r . G I F D(R)$.

(2) $\sup \left\{f d_{R}(M) \mid M\right.$ is a Gorenstein injective left $R$-module $\}$.

(3) $\sup \left\{f d_{R}(M) \mid M\right.$ is a left $R$-module with $\left.\operatorname{Gid}_{R}(M)<\infty\right\}$.

(4) $\sup \left\{f d_{R}\left(N^{+}\right) \mid N\right.$ is a right $R$-module with $\left.\operatorname{Gfd}_{R}(N)<\infty\right\}$.

(5) $\sup \left\{i d_{R}(N) \mid N\right.$ is a Gorenstein flat right $R$-module $\}$.

(6) $\sup \left\{i d_{R}(N) \mid N\right.$ is a right $R$-module with $\left.\operatorname{Gfd}_{R}(N)<\infty\right\}$.

Proof. Since $n$-Gorenstein rings are Noetherian, this result follows immediately by Theorem 3.8 and Proposition 3.9.

It has been shown that a commutative ring $R$ is an IF ring if and only if $\operatorname{Hom}_{R}(A, B)$ is injective for all injective $R$-modules $A$ and $B$ ([6, Corollary $3.22])$. Now we have the following results.

Theorem 3.11. Let $R$ be a commutative ring and $n$ a nonnegative integer. Then the following are equivalent:

(1) $G I F D(R) \leqslant n$. 
(2) $i d_{R} \operatorname{Hom}_{R}(A, B) \leqslant n$ for all Gorenstein injective $R$-modules $A$, all injective $R$-modules $B$.

Moreover, if $R$ is commutative Artinian, then the above conditions are equivalent to

(3) $f d_{R}\left(A \otimes_{R} F\right) \leqslant n$ for all Gorenstein injective $R$-modules $A$ and all flat $R$-modules $F$.

Proof. (1) $\Rightarrow(2)$ Let $A$ be a Gorenstein injective $R$-module and $B$ an injective $R$-module. Then $\operatorname{fd}_{R}(A) \leqslant n$ by $(1)$, and hence we have a flat resolution of $A$

$$
0 \rightarrow F_{n} \rightarrow F_{n-1} \rightarrow \cdots \rightarrow F_{0} \rightarrow A \rightarrow 0,
$$

which gives rise to the exactness of the sequence

$$
0 \rightarrow \operatorname{Hom}_{R}(A, B) \rightarrow \operatorname{Hom}_{R}\left(F_{0}, B\right) \rightarrow \cdots \rightarrow \operatorname{Hom}_{R}\left(F_{n}, B\right) \rightarrow 0 .
$$

Since $F_{i}(0 \leqslant i \leqslant n)$ is flat and $B$ is injective, $\operatorname{Hom}_{R}\left(F_{i}, B\right)$ is injective by $[19$, Theorem 3.44]. Consequently, $\operatorname{id}_{R} \operatorname{Hom}_{R}(A, B) \leqslant n$, and so (2) holds.

$(2) \Rightarrow(1)$ Let $A$ be a Gorenstein injective $R$-module, and let

$$
\cdots \rightarrow F_{n} \rightarrow F_{n-1} \rightarrow \cdots \rightarrow F_{0} \rightarrow A \rightarrow 0
$$

be a flat resolution of $A$. Let $K=\operatorname{im}\left(F_{n} \rightarrow F_{n-1}\right)$. Then we get the exact sequence

$$
0 \rightarrow K \rightarrow F_{n-1} \rightarrow \cdots \rightarrow F_{0} \rightarrow A \rightarrow 0 .
$$

For any injective $R$-module $B$, the sequence

$$
0 \rightarrow \operatorname{Hom}_{R}(A, B) \rightarrow \operatorname{Hom}_{R}\left(F_{0}, B\right) \rightarrow \cdots \rightarrow \operatorname{Hom}_{R}(K, B) \rightarrow 0
$$

is exact. Since $\operatorname{id}_{R} \operatorname{Hom}_{R}(A, B) \leqslant n$ and each $\operatorname{Hom}_{R}\left(F_{i}, B\right)(0 \leqslant i \leqslant n-1)$ is injective, we have $\operatorname{Hom}_{R}(K, B)$ is injective for any injective $R$-module $B$. So $K$ is flat by [14, Proposition 11.35]. Thus $\operatorname{fd}_{R}(A) \leqslant n$, and hence (1) follows by Theorem 3.4.

$(1) \Rightarrow(3)$ Suppose $R$ is a commutative Artinian ring. If $A$ is Gorenstein injective and $F$ is flat, then $A \otimes_{R} F$ is Gorenstein injective by [22, Corollary 2.17]. The desired result follows from (1).

$(3) \Rightarrow$ (1) follows by taking $F=R$. This completes the proof.

Now we give some properties of rings with small global GI-flat dimension.

Proposition 3.12. The following are equivalent for any ring $R$.

(1) $r . G I F D(R) \leqslant 1$

(2) Every submodule of a projective right $R$-module is GI-flat.

(3) Every submodule of a flat right $R$-module is GI-flat.

(4) Every submodule of a GI-flat right $R$-module is GI-flat.

Proof. (1) $\Rightarrow(4)$ Let $N$ be a submodule of a GI-flat right $R$-module $M$. Then there is an exact sequence $0 \rightarrow N \rightarrow M \rightarrow M / N \rightarrow 0$. For any Gorenstein injective left $R$-module $G$, we have the exactness of

$$
\operatorname{Tor}_{2}^{R}(M / N, G) \rightarrow \operatorname{Tor}_{1}^{R}(N, G) \rightarrow \operatorname{Tor}_{1}^{R}(M, G) .
$$


The first term is zero since $\mathrm{fd}_{R}(G) \leqslant 1$, and the last term is zero since $M$ is GI-flat. Consequently, $\operatorname{Tor}_{1}^{R}(N, G)=0$, and hence (4) holds.

$(4) \Rightarrow(3) \Rightarrow(2)$ is trivial.

$(2) \Rightarrow(1)$ Let $G$ be a Gorenstein injective left $R$-module. For any right $R$-module $M$, there is an exact sequence $0 \rightarrow K \rightarrow P \rightarrow M \rightarrow 0$ with $P$ projective. Thus we get the exactness of $0 \rightarrow \operatorname{Tor}_{2}^{R}(M, G) \rightarrow \operatorname{Tor}_{1}^{R}(K, G)$. The last term is zero by $(2)$. Therefore $\operatorname{Tor}_{2}^{R}(M, G)=0$, which implies $\operatorname{fd}_{R}(G) \leqslant 1$, and so (1) follows.

Corollary 3.13. If $R$ is a ring with r.GIFD $(R) \leqslant 1$, then every GI-flat right $R$-module is strongly GI-flat.

Proof. Let $M$ be a GI-flat right $R$-module. Then there is an exact sequence $0 \rightarrow K \rightarrow P \rightarrow M \rightarrow 0$ with $P$ projective. Thus $K$ is GI-flat by Proposition 3.12 since $\operatorname{r.GIFD}(R) \leqslant 1$, and so $M$ is strongly GI-flat by induction.

It is well known that a left coherent ring $R$ is left semihereditary if and only if $w D(R) \leqslant 1$. Next we give a characterization of such rings.

Proposition 3.14. The following are equivalent for a left coherent ring $R$.

(1) $R$ is left semihereditary.

(2) r.GIFD $(R) \leqslant 1$ and every GI-flat right $R$-module is flat.

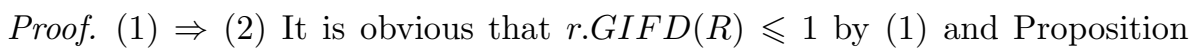
3.5. Let $M$ be a GI-flat right $R$-module. For any left $R$-module $N$, there is an exact sequence $0 \rightarrow N \rightarrow E \rightarrow L \rightarrow 0$ with $E$ injective. Thus we get the exactness of

$$
\operatorname{Tor}_{2}^{R}(M, L) \rightarrow \operatorname{Tor}_{1}^{R}(M, N) \rightarrow \operatorname{Tor}_{1}^{R}(M, E) .
$$

The first term is zero by (1), and the last term is zero since $M$ is GI-flat. So $\operatorname{Tor}_{1}^{R}(M, N)=0$ for all left $R$-modules $N$, and hence $M$ is flat.

$(2) \Rightarrow(1)$ Suppose the condition (2) holds. Let $M$ be a right $R$-module and $0 \rightarrow K \rightarrow F \rightarrow M \rightarrow 0$ be exact with $F$ flat. By Proposition $3.12, K$ is GI-flat, and so it is flat by $(2)$. Therefore $\operatorname{fd}_{R}(M) \leqslant 1$, which implies that $w D(R) \leqslant 1$, and so $R$ is left semihereditary.

Recall that a left $R$-module $C$ is said to be cotorsion [21] provided that $\operatorname{Ext}_{R}^{1}(F, C)=0$ for all flat left $R$-modules $F$; a submodule $T$ of a left $R$ module $N$ is called a pure submodule [11] if $0 \rightarrow A \otimes_{R} T \rightarrow A \otimes_{R} N$ is exact for every right $R$-module $A$; an exact sequence $0 \rightarrow T \rightarrow N \rightarrow N / T \rightarrow 0$ is called pure exact if $T$ is a pure submodule of $N$. An $R$-module $M$ is said to be pure injective [11] if for every pure exact sequence $0 \rightarrow T \rightarrow N$ of $R$-modules, $\operatorname{Hom}_{R}(N, M) \rightarrow \operatorname{Hom}_{R}(T, M) \rightarrow 0$ is exact.

Theorem 3.15. The following assertions are equivalent for any ring $R$ :

(1) Every right $R$-module is GI-flat.

(2) Every Gorenstein injective left $R$-module is flat.

(3) Every cotorsion left $R$-module is GI-injective. 
(4) Every pure injective left $R$-module is GI-injective.

(5) Every finitely presented right $R$-module is GI-flat.

Proof. $(1) \Leftrightarrow(2)$ is by definition.

$(2) \Rightarrow(3)$ Let $M$ be a cotorsion left $R$-module. For any Gorenstein injective left $R$-module $N$, it follows that $N$ is flat by (2). Consequently $\operatorname{Ext}_{R}^{1}(N, M)=$ 0 , and so $M$ is GI-injective.

$(3) \Rightarrow(4)$ holds since every pure injective module is cotorsion.

$(4) \Rightarrow(1)$ Let $M$ be a right $R$-module. Then $M^{+}$is pure injective by [11, Proposition 5.3.7], and so $M^{+}$is GI-injective by (4). It follows that $M$ is GI-flat.

$(1) \Rightarrow(5)$ is trivial.

$(5) \Rightarrow(2)$ Let $E$ be a Gorenstein injective left $R$-module. Then $\operatorname{Tor}_{1}^{R}(M, E)$ $=0$ for any finitely presented right $R$-module $M$ by (5). Therefore $E$ is flat, as desired.

Proposition 3.16. Let $R$ be a commutative ring. Then each Gorenstein injective $R$-module is flat if and only if $R$ is von Neumann regular.

Proof. "If" part is trivial.

"Only if" part. Suppose that every Gorenstein injective $R$-module is flat. Then $R$ is an IF ring since every injective module is Gorenstein injective. So $R$ is an FC ring by [6, Corollary 3.14]. Let $M$ be an $R$-module. Then $M$ is Gorenstein flat by $[8$, Theorem 6$]$. It follows from $\left[17\right.$, Theorem 3.6] that $M^{+}$ is Gorenstein injective, and so $M^{+}$is flat by the hypothesis. Consequently, $M$ is FP-injective by [15, Theorem 2.2] since $R$ is coherent. This proves that every $R$-module is FP-injective. Thus $R$ is von Neumann regular.

For the case $n=0$ in Theorem 3.11, we obtain the following characterizations of von Neumann regular rings.

Corollary 3.17. The following are equivalent for a commutative ring $R$.

(1) $R$ is von Neumann regular.

(2) For every Gorenstein injective $R$-module $A, \operatorname{Hom}_{R}(A, B)$ is injective for all injective $R$-modules $B$.

Proposition 3.18. Let $R$ be any ring. Then $R$ is an IF ring if and only if every Gorenstein injective $R$-module (left and right) is Gorenstein flat.

Proof. Let $R$ be an IF ring and $M$ a Gorenstein injective left $R$-module. There is an exact sequence of injective left $R$-modules

$$
\mathbf{E}=\cdots \rightarrow E_{1} \rightarrow E_{0} \rightarrow E^{0} \rightarrow E^{1} \rightarrow \cdots
$$

with $M=\operatorname{ker}\left(E^{0} \rightarrow E^{1}\right)$. Then $\mathbf{E}$ is also an exact sequence of flat left $R$ modules since $R$ is IF. Next it suffices to show that $I \otimes_{R} \mathbf{E}$ is exact for any injective right $R$-module $I$. The result follows immediately since $I$ is flat. So every Gorenstein injective left $R$-module is Gorenstein flat. The case of right $R$-modules can be proved similarly. 
Conversely, let $E$ be any injective left $R$-module. Then $E$ is Gorenstein flat since every injective module is Gorenstein injective. There exists an exact sequence $0 \rightarrow E \rightarrow F \rightarrow L \rightarrow 0$ with $F$ flat. Since $E$ is injective, the sequence $0 \rightarrow E \rightarrow F \rightarrow L \rightarrow 0$ splits. So $E$ is flat as a direct summand of $F$. Thus $R$ is left IF. Similarly, $R$ is right IF. Consequently $R$ is IF.

Acknowledgements. This research was partially supported by NSFC(No. 11171240 and 11226057), and the Scientific Research Foundation of CUIT(No. KYTZ201201 and J201217). I would like to thank the referee for his/her helpful comments, which have improved the article.

\section{References}

[1] D. Bennis, A note on Gorenstein flat dimension, Algebra Colloq. 18 (2011), no. 1, $155-161$.

[2] D. Bennis and N. Mahdou, Strongly Gorenstein projective, injective, and flat modules, J. Pure Appl. Algebra 210 (2007), no. 2, 437-445.

[3] H. Cartan and S. Eilenberg, Homological Algebra, Princeton University Press, 1956.

[4] L. W. Christensen, Gorenstein Dimensions, Lecture Notes in Math., 1747, SpringerVerlag, Berlin, 2000.

[5] R. R. Colby, Rings which have flat injective modules, J. Algebra 35 (1975), 239-252.

[6] N. Q. Ding and J. L. Chen, The flat dimensions of injective modules, Manuscripta Math. 78 (1993), no. 2, 165-177.

[7] - On copure flat modules and flat resolvents, Comm. Algebra 24 (1996), no. 3, 1071-1081.

[8] _ Coherent rings with finite self-FP-injective dimension, Comm. Algebra 24 (1996), no. 9, 2963-2980.

[9] E. E. Enochs and O. M. G. Jenda, Copure injective resolutions, flat resolvents and dimensions, Comment. Math. Univ. Carolin. 34 (1993), no. 2, 203-211.

[10] _ Gorenstein injective and projective modules, Math. Z. 220 (1995), no. 4, 611633.

[11] Relative Homological Algebra, Walter de Gruyter, Berlin, 2000.

[12] E. E. Enochs, O. M. G. Jenda, and J. A. López-Ramos, The existence of Gorenstein flat covers, Math. Scand. 94 (2004), no. 1, 46-62.

[13] E. E. Enochs, O. M. G. Jenda, and B. Torrecillas, Gorenstein flat modules, Nanjing Daxue Xuebao Shuxue Bannian Kan 10 (1993), no. 1, 1-9.

[14] C. Faith, Algebra I: Rings, Modules and Categories, Springer, Berlin-Heidelberg-New York, 1981.

[15] D. J. Fieldhouse, Character modules, dimension and purity, Glasgow Math. J. 13 (1972), 144-146.

[16] Z. H. Gao, On GI-injective modules, Comm. Algebra 40 (2012), no. 10, 3841-3858.

[17] H. Holm, Gorenstein homological dimensions, J. Pure Appl. Algebra 189 (2004), no. 1-3, 167-193.

[18] L. X. Mao and N. Q. Ding, Gorenstein FP-injective and Gorenstein flat modules, J. Algebra Appl. 7 (2008), no. 4, 491-506.

[19] J. J. Rotman, An Introduction to Homological Algebra, Academic Press, 1979.

[20] R. Sazeedeh, Strongly torsion free, copure flat and Matlis reflexive modules, J. Pure Appl. Algebra 192 (2004), no. 1-3, 265-274.

[21] J. Z. Xu, Flat Covers of Modules, Lecture Notes in Math., 1634, Springer-Verlag, Berlin, 1996. 
[22] X. Y. Yang and Z. K. Liu, Strongly Gorenstein projective, injective and flat modules, J. Algebra 320 (2008), no. 7, 2659-2674.

College of Mathematics

Chengdu University of Information Technology

Sichuan 610225, P. R. China

E-mail address: gaozenghui@cuit.edu.cn 